\title{
Welches Potenzial haben virtuelle Realitäten in der klinischen und forensischen Psychiatrie? Ein Überblick über aktuelle Verfahren und Einsatzmöglichkeiten
}

\author{
Hermann Barbe ${ }^{1}$ Bruno Siegel ${ }^{1}$ Jürgen L. Müller ${ }^{1}$ Peter Fromberger ${ }^{1}$ \\ Eingegangen: 13. Mai 2020 / Angenommen: 2. Juni 2020 / Online publiziert: 2. Juli 2020 \\ (c) Der/die Autor(en) 2020
}

\section{Zusammenfassung}

Virtuelle Realitäten (VR) werden in der Diagnose und Behandlung von Patienten im klinischen Feld bereits seit 20 Jahren erfolgreich eingesetzt und weiterentwickelt. Seit etwas mehr als 5 Jahren gibt es nun auch erste Beispiele über die Anwendung von VR in psychiatrisch-forensischen Kontexten. Für die forensische Psychiatrie ist die Möglichkeit, realistische, sichere und kontrollierbare Diagnostik- und Lernumgebungen zu schaffen, der ausschlaggebende Vorteil der VR-Technologie. So können z.B. Straftäter in Szenarien behandelt oder begutachtet werden, welche im echten Leben risikoreich, unethisch oder ökologisch invalide wären. In diesem Artikel werden unterschiedliche aktuelle Studienbeispiele zu klinischer Behandlung und Diagnose von Patienten sowie der forensischen Prognose und Therapie von Straftätern vorgestellt. Damit zeigt der Überblick, dass VR mittlerweile auch in der forensischen Psychiatrie ein vielversprechendes Werkzeug sein kann, welches bereits etablierte Instrumente ergänzen oder erweitern kann. Auch in der Ausbildung von forensisch-psychiatrischem Fachpersonal können VR-Anwendungen eine Hilfe sein. Hier gibt es bereits erste vielversprechende Einsätze durch das Training mithilfe von virtuellen Patienten, jedoch benötigt es noch umfangreiche Forschungsarbeit auf diesem Feld, um sie im professionellen Alltag einsetzen zu können. Vor dem Einsatz von VR-Anwendungen sollten sich Forscher und Praktiker neben den Vorteilen auch mit den Nachteilen von VR auseinandersetzen und ein besonderes Augenmerk auf die ethischen Richtlinien werfen, welche in den letzten Jahren dazu erarbeitet wurden. Die stetige Weiterentwicklung und der immer breitere Einsatz von VR im klinischen und forensisch-psychiatrischen Feld zeigen, dass VR auch hier das Potenzial hat, ein etabliertes Forschungs- sowie Therapieinstrument zu werden.

Schlüsselwörter Diagnostik · Therapie · Virtuelle Simulationspatienten · Prognose

Hermann Barbe, Psychologe M.Sc.

hermann.barbe@med.uni-goettingen.de

1 Klinik für Psychiatrie und Psychotherapie, Forensische Psychiatrie, Universitätsmedizin Göttingen, Göttingen, Deutschland 


\title{
What is the potential of virtual reality in clinical and forensic psychiatry? An overview of current procedures and possible applications
}

\begin{abstract}
Virtual reality (VR) applications have already been successfully used in the diagnostics and treatment of psychiatric disorders for 20 years. After approximately 5 years there are now the first examples of the application of VR in the context of forensic psychiatry. The most important advantage of VR for forensic applications is the possibility to develop realistic, safe and highly controllable virtual diagnostics and learning environments. In this way practitioners can treat or assess criminal offenders in scenarios that would otherwise be risky, unethical or ecologically invalid. This article presents various study examples of current applications of VR for clinical treatment and diagnosis of patients as well as examples for the prognosis and therapy of criminal offenders. This overview shows that VR can now also be a promising tool in forensic psychiatry, which can supplement or extend already established instruments. Applications of VR can also be helpful in the training of forensic psychiatric personnel, as can be seen in some promising results of training studies with virtual patients; however, before they can be implemented into routine professional life, extensive research is needed in this field. Before the introduction of VR applications in research and practice, not only the advantages but also the disadvantages of VR must be taken into consideration and special attention should be paid to the ethical guidelines, which have been developed for this purpose. The continuous development and the increasingly widespread use of VR in clinical and forensic psychiatry imply that VR also has the potential to become an established instrument in research and therapy.
\end{abstract}

Keywords Diagnostics $\cdot$ Psychotherapy $\cdot$ Virtual standardized patients $\cdot$ Prognosis

If I can see it and believe it, then I can achieve it

(A. Schwarzenegger)

\section{Einführung}

Virtuelle Realitäten (VR) sind in der psychologischen und psychiatrischen Forschung sowie in der Psychotherapie schon lange keine ungewöhnliche Anwendung mehr. Bereits seit 20 Jahren wird eine Vielzahl von VR-Anwendungen z.B. für die Diagnostik und Behandlung von Phobien (Côté und Bouchard 2005), posttraumatischen Belastungsstörungen (Reger et al. 2011) oder Suchterkrankungen (Cho et al. 2008) erfolgreich eingesetzt und weiterentwickelt. Dabei haben insbesondere die technischen Fortschritte der Hersteller von VR-Brillen (,head mounted displays", HMD) in den letzten 5 Jahren für eine starke Zunahme an Vielfalt neuer Einsatzmöglichkeiten geführt (Bailenson 2018), welche nun auch häufiger außerhalb von Forschung in Medizin, Architektur, Sport, körperlicher Rehabilitation und Kunst oder beim Militär, zu finden sind (Cornet und Van Gelder 2020). Verglichen mit den Fortschritten in anderen Forschungsbereichen, steckte die Anwendung von VR in der forensischen Psychologie und Psychiatrie bis vor wenigen Jahren in den Kinderschuhen (Benbouriche et al. 2014). Anhand von Beispielen aus der Forschungs- sowie Praxisanwendung in der klinischen sowie forensischen Psychologie und Psychiatrie sollen das Potenzial für zukünftige Forschung aufgezeigt und wichtige ethische und praktischen Überlegungen für den Einsatz von
VR diskutiert werden. Zunächst ist es jedoch wichtig, zum Verständnis der Beispiele die wichtigsten technologischen Begrifflichkeiten zu erläutern.

\section{Begrifflichkeiten}

Bevor man von einer VR sprechen kann, müssen mehrere Komponenten zur Verfügung stehen. Zunächst braucht man eine virtuelle Umgebung. Diese besitzt spezifische sensorische Stimuli, welche bei einer Wahrnehmung durch eine Person bei ihr den Eindruck verschaffen, die virtuelle Umgebung könnte auch ein reales, nichtkünstliches, Erlebnis sein (Ryan et al. 2019). Legt man sich diese Definition zugrunde, wird deutlich, dass dieser Effekt nicht ausschließlich mittels visueller Stimuli erzielt werden muss, sondern prinzipiell auch durch haptische oder akustische virtuelle Umgebungen erreicht werden kann. Darüber hinaus braucht man eine zumeist technische Komponente, mithilfe derer man die virtuelle Umgebung gestalten und sie dem Nutzer präsentieren kann. In diesem Artikel werden computergenerierte virtuelle Umgebungen fokussiert. Mittlerweile konnte eine Vielzahl unterschiedlicher Forschungsansätze zeigen, dass solche virtuellen Umgebungen sehr gut in der Lage sind, in dem Nutzer Sinneserfahrungen, Emotionen und Interaktionen so zu evozieren, dass er sie nicht von realen Erlebnissen unterscheiden kann (Parsons et al. 2017). Je mehr und je stärker dabei Sinnesmodalitäten des Nutzers aktiviert werden, umso mehr spricht man von einer immersiven virtuellen Realität (Bohil et al. 2011). Immersion ist dabei kein psychologischer Zustand, sondern beschreibt 
die Eigenschaften des für die VR eingesetzten Systems (Bouchard und Rizzo 2019). Der Grad der Immersion lässt sich durch z.B. unterschiedliche technische Darstellungsformen (Monitor vs. HMD), unterschiedliche Auflösungen oder die Erfassung von Kopfbewegungen des Nutzers und deren Übersetzung in die VR manipulieren (Fromberger et al. 2018a). So lassen sich VR-Anwendungen auf einem Kontinuum von schwach immersiv (z. B. Präsentation einer virtuellen Umgebung über einen Monitor) bis stark immersiv (z.B. Präsentation einer virtuellen Umgebung über ein HMD) einordnen (Milgram und Kishino 1994). Zwischen diesen beiden Polen liegen Anwendungen, welche sich der „mixed reality“ bzw. ,augmented reality“ zuordnen lassen. Dabei werden virtuelle Objekte über z. B. ein Handydisplay mit realen Umgebungen vermischt.

Je glaubhafter eine virtuelle Umgebung einem Nutzer erscheint, umso größer ist die Wahrscheinlichkeit, dass sich ein sog. Präsenzerleben einstellt. Präsenz kann als eine Art Wahrnehmungstäuschung verstanden werden, welche aus der Illusion der Plausibilität (Objekte in der VR machen einen realen Eindruck) sowie der Illusion des Orts (Nutzer haben den Eindruck, ,in“ der virtuellen Umgebung zu sein und nicht am tatsächlichen Einsatzort) entsteht (Schuemie et al. 2004; Slater 2009). Daneben gibt es das soziale Präsenzerleben. Hierunter versteht man die Plausibilität der sozialen Interaktion mit einer in der virtuellen Realität dargestellten Figur, also inwiefern ein Nutzer das Gefühl hat, die virtuelle Figur könnte real sein (Alsina-Jurnet et al. 2011). Das Erzeugen von Präsenz und sozialer Präsenz gehört zu den wichtigsten Eigenschaften von VR sowohl im Forschungs- als auch im Anwendungskontext. Denn sie sorgen dafür, dass Nutzer in immersiven virtuellen Umgebungen emotionale und behaviorale Reaktionen zeigen, welche den in realen Situationen auftretenden entsprechen (Alsina-Jurnet et al. 2011). Dies erlaubt es Forschern, reale Situationen, deren Implementierungen vielleicht aus ethischen (Konfrontation von Straftätern mit vulnerablen Dritten; z. B. Fromberger et al. 2018b) oder praktischen Gesichtspunkten (Behandlung einer Flugangst in einem echten Flugzeug; z. B. Mühlberger et al. 2005) kaum umsetzbar oder sogar völlig unmöglich (Transformationen in einen Körper mit anderer Hautfarbe oder anderen Geschlecht; Groom et al. 2009) sind, in einem standardisierten virtuellen Umfeld zu simulieren. Virtuelle-Realitäts-Anwendungen können so auch die ökologische Validität und damit die Generalisierbarkeit von psychologischen und psychiatrischen Diagnostik- oder Therapierverfahren steigern, indem sie reale Lebenssituationen besser nachahmen können als herkömmliche Methoden (Fromberger et al. 2018a).

\section{VR-Anwendungen in der Psychotherapie und klinischen Diagnostik}

Im Bereich der Psychotherapie spielen VR-Anwendungen insbesondere im Rahmen klassisch verhaltenstherapeutisch ausgelegter Expositionstherapien eine große Rolle. Diese sind v. a. bei der Behandlung von Angsterkrankungen nachweislich wirksam (Craske et al. 2018), und der zugrunde liegende Prozess der wiederholten Konfrontation mit angstbesetzten Stimuli lässt sich gut auf virtuelle Objekte und Umgebungen übertragen. Die oben genannte Ähnlichkeit der emotionalen und behavioralen Reaktionen des Anwenders in virtuellen Umgebungen im Vergleich zur entsprechenden realen Situation ermöglicht dementsprechend eine Behandlung analog zur klassischen Therapie in vivo. So konnte etwa gezeigt werden, dass zwangserkrankte Probanden mit Kontaminationsängsten in der virtuellen Umgebung einer stark verschmutzten öffentlichen Toilette die zu erwartenden Angstreaktionen zeigen (Laforest et al. 2016). Virtuelle-Realität-basierte Expositionstherapien demonstrieren bei der Behandlung von Angsterkrankungen vergleichbare Effekte wie In-vivo-Therapien (Botella et al. 2017; Carl et al. 2019), werden jedoch auch zunehmend erfolgreich zur Behandlung weiterer psychiatrischer Krankheitsbilder genutzt, wie etwa der posttraumatischen Belastungsstörung, Schizophrenie oder von Suchterkrankungen (für eine Übersicht: Park et al. 2019). Auch im Bereich psychiatrischer Diagnostik kann der Einsatz von VR-Anwendungen Vorteile mit sich bringen, da innerhalb der VR Situationen, in denen relevante Symptome auftreten, kontrolliert simuliert werden können. Auf diese Weise werden Störfaktoren wie lückenhafte Erinnerungen oder mangelnde Objektivität durch Diagnostiker reduziert (Freeman et al. 2017). Vielversprechende Ergebnisse beim Einsatz von VR als diagnostisches Instrument gibt es bereits beispielsweise bei der Erkennung einer leichten kognitiven Beeinträchtigung oder Alzheimer-Krankheit (für einen Überblick: Clay et al. 2020) sowie sozialer Phobie (Dechant et al. 2017).

\section{VR-Anwendungen in der forensischen Psychiatrie}

Trotz dieser Erfolge beim Einsatz von VR bei der Diagnostik und Therapie psychischer Erkrankungen wurden bislang nur wenige Studien zur möglichen Anwendung im forensisch-psychiatrischen Kontext durchgeführt. Die Idee, deviantes sexuelles Interesse mittels VR-Anwendungen zu erfassen, wurde erstmals von Laws und Gress (2004) erwähnt. Zwei weitere Überblicksarbeiten (Benbouriche et al. 2014; Fromberger et al. 2014) befassten sich mit dem Potenzial von VR-Anwendungen für die Diagnostik und Therapie psychisch kranker Straftäter wie beispielsweise bei Kin- 
desmissbrauchstätern. Die Autoren nennen dabei, neben der hohen ökologischen Validität und der guten Kontrollierbarkeit virtueller Situationen, weitere Vorteile von VRAnwendungen für den forensisch-psychiatrischen Bereich: VR-Anwendungen erlauben es, Straftäter mit Situationen, welche störungsrelevantes Verhalten hervorrufen können, ohne Gefährdung Dritter zu konfrontieren. Dadurch können neue Fähigkeiten und Verhaltensweisen in rückfallkritischen virtuellen Situationen eingeübt werden (Fromberger et al. 2014, 2018b). Die Möglichkeit, realistische physische, soziale und emotionale Stimuli innerhalb der VR-Anwendung zu präsentieren, erlaubt es beispielsweise, die Selbstregulationsfähigkeiten von Straftätern anzusprechen. Dies könnte ebenfalls ein spezifischer Vorteil der Anwendung von VR in der forensischen Psychiatrie sein, da Selbstregulationsfähigkeiten eine wichtige Rolle für das Verhalten von Straftätern spielen und so eine Einschätzung dieser ermöglicht wird (Benbouriche et al. 2014). Perspektivisch sind auch therapeutische Interventionen, welche auf Selbstregulation abzielen, denkbar. So wurden in jüngerer Zeit beispielsweise VR-gestützte Interventionen zur Reduktion aggressiven Verhaltens sowohl in stationären als auch ambulanten forensischen Kontexten getestet: So entwickelten und testeten Klein Tuente et al. (2019) eine VR-basierte Intervention zur Reduktion aggressiven Verhaltens an 128 forensischen Patienten in stationärer Behandlung. In der randomisierten kontrollierten Studie zeigte sich keine Reduktion aggressiven Verhaltens, allerdings ergaben sich Hinweise auf eine kurzfristige Verbesserung damit assoziierter Aspekte wie Impulsivität oder Feindseligkeit. Eine weitere Studie untersucht aktuell ebenfalls die Möglichkeit, Aggressivität und Wut mittels einer VR-basierten Intervention zu verringern (Smeijers und Koole 2019). Anhand einer Stichprobe von 60 ambulant behandelten forensisch-psychiatrischen Patienten soll gezeigt werden, dass das Einüben von Vermeidungsbewegungen in Reaktion auf wütende Gesichter zu einer Verringerung von Aggressivität führt. Ein anderer Ansatz wurde von Seinfeld et al. genutzt (2018), welche die Täter häuslicher Gewalt mittels VR-Anwendung in den Körper einer Frau schlüpfen und einen Übergriff erleben ließen. Die angestrebte Reduktion aggressiven Verhaltens sollte in diesem Fall durch die Verbesserung von Fähigkeiten zu Empathie und Perspektivwechsel erreicht werden. An einer Stichprobe von 20 Straftätern und 19 Kontrollprobanden konnte gezeigt werden, dass durch diesen Perspektivwechsel die Fähigkeit, ängstliche Gesichtsausdrücke bei Frauen zu erkennen, in der Gruppe der Straftäter anstieg.

Ein weiterer klinischer Einsatzbereich ist die Nutzung von VR bei der Diagnostik devianter sexueller Interessen, insbesondere derer von Kindesmissbrauchstätern (Renaud et al. 2009; Benbouriche et al. 2014; Fromberger et al. 2014). So konnten etwa Renaud et al. (2014) zeigen, dass hoch immersive visuelle Stimuli effektiver sexuelle Erre- gung hervorrufen können als auditorische Stimuli, wobei sexuelle Erregung mittels Penisplethysmographie erfasst wurde. Bei dem Vergleich von 22 Kindesmissbrauchstätern und 42 gesunden Männern führte die Präsentation der computergenerierten Charaktere in VR zu einer signifikant höheren Klassifizierungsgenauigkeit hinsichtlich des sexuellen Interesses der Probanden. Dass VR prinzipiell geeignet ist, sexuelles Interesse zu erfassen, zeigen Studien aus dem nichtforensischen Bereich mit Normalprobanden. So verglichen Fromberger et al. (2015) ebenfalls hochimmersive und in VR präsentierte Stimuli mit weniger immersiven Reizen, welche auf einem Bildschirm präsentiert wurden. Dabei sollten 22 homosexuelle und 25 heterosexuelle Probanden die dargestellten virtuellen Charaktere hinsichtlich ihrer sexuellen Attraktivität bewerten. Als Maß für sexuelles Interesse wurde hier jedoch ohne das Wissen der Probanden die „viewing time“, die Zeit, welche zwischen Beginn der Präsentation des Stimulus und Abgabe der Bewertung verstrich, erhoben. Auch hier zeigte sich in der VR-Bedingung eine Verbesserung der Klassifikationsgenauigkeit. Eine weitere Möglichkeit, sexuelles Interesse mittels VR-Technologie zu erfassen, scheint die Nutzung eines Annäherungs-Vermeidungs-Paradigmas zu sein. So instruierten Welsch et al. (2020) 48 gynäphile und 24 androphile männliche Probanden dazu, in Abhängigkeit vom Geschlecht eines virtuellen Charakters eine Bewegung auf diesen zu oder von diesem weg auszuführen. In einem zweiten Experiment sollten sie sich den Figuren dann so nähern, als wollten sie ein Gespräch mit diesen beginnen. Die Analyse der Bewegungsdaten legte nahe, dass wahrgenommene sexuelle Attraktivität der Stimuli die Annäherungsreaktion erleichterte und mit einer geringeren bevorzugten interpersonalen Distanz zusammenhing. Oben genannte Studien zeigen, dass die Verwendung von VRAnwendungen die Salienz sexueller Stimuli erhöhen kann. Dementsprechend erscheint eine Nutzung zur Erfassung devianter sexueller Interessen vielversprechend.

Auch die Risikoeinschätzung von Straftätern könnte vom Einsatz VR-basierter Verfahren profitieren. Dem liegt insbesondere die Möglichkeit, Straftäter ohne Gefährdung der Öffentlichkeit in einer kontrollierten Umgebung störungsrelevanten Stimuli auszusetzen, zugrunde. So ließen Fromberger et al. (2018b) in einer weiteren Studie 6 Kindesmissbrauchstäter und 7 gesunde Probanden virtuelle Risikosituationen durchlaufen, in welchen sie von Kindern angesprochen wurden. Ziel war es zu überprüfen, ob im Rahmen der Therapie erarbeitete Strategien zum Umgang mit solchen Situationen unter realistischen Bedingungen auch tatsächlich abgerufen werden können. Obwohl weitergehende Studien nötig sind, zeigt sich auch hier, dass der Einsatz von VR in diesem Bereich vollkommen neue Möglichkeiten eröffnen könnte. So konnte beispielsweise gezeigt werden, 
dass nur jeder 2. Straftäter die in der Therapie besprochenen Coping-Skills auch in der virtuellen Risikosituation zeigte.

\section{Training von forensisch-psychiatrischem Fachpersonal durch virtuelle Simulationspatienten}

Bereits Fromberger et al. (2014) wiesen auf das hohe Potenzial von VR für das Training von Fachpersonal im forensisch-psychiatrischen Kontext hin. Gesprächstechniken, Anamnesedurchführung, Erkennen von Symptomen, Diagnosestellung, zwischenmenschliche Kommunikation: Das alles sind Fähigkeiten, die während einer medizinischen oder klinisch-psychologischen Ausbildung vermittelt werden sollten. Auch in weiteren Berufsfeldern, wie bei der Polizei, bei Juristen oder bei forensisch-psychiatrischen Gutachtern gehören sie zum beruflichen Alltag. Trainiert werden solche Fähigkeiten bereits seit den 1960er-Jahren v.a. im medizinischen Bereich mithilfe von Simulationspatienten (Artstein et al. 2008) - Schauspielern, die versuchen, Patienten oder Zeugen so realitätsgetreu wie möglich darzustellen. Obwohl der Einsatz von Simulationspatienten Vorteile gegenüber herkömmlichen Instruktionsmethoden hat (Benedict 2010), gibt es auch einige Nachteile: Zum einen ist deren Einsatz teuer, sodass ein „Eins-zu-Eins“ beim Training von Gruppen kaum möglich ist (weshalb stattdessen häufiger die Seminarteilnehmer selber in Rollenspielen zwischen der Befrager- und Patienten-/Zeugenrolle hin und herwechseln). Zum anderen ist es häufig schwer, eine ausreichende Anzahl an (professionellen) Schauspielern zu finden, die bestimmte relevante Bevölkerungsgruppen repräsentieren, wie ältere Menschen für die Medizin oder Kinder im forensischen Kontext einer Zeugenbefragung. Abschließend sind auch standardisierte Trainingsdurchläufe zwischen verschiedenen Simulationspatienten nur schwer umsetzbar, da auch professionelle Schauspieler nicht wiederholt das exakt gleiche Verhalten simulieren können. Deshalb werden seit etwa 15 Jahren sowohl in der Medizin als auch in der psychologischen Forschung sog. virtuelle Simulationspatienten (VSP) eingesetzt. Dabei werden computergenerierte realistische virtuelle Charaktere genutzt, die, je nach Trainingsart, unterschiedliche klinische Fälle oder Personengruppen repräsentieren können (Hubal et al. 2000). Die Vorteile solcher VSP sind v. a. die Möglichkeit, immer wieder bestimmte Verhaltensweisen üben zu können, die Schwierigkeit individuell an den Nutzer anzupassen, standardisiert vorgehen und ethische Konflikte bei der Befragung bestimmter Gruppen (z.B. Kinder im Kontext sexuellen Missbrauchs) vermeiden zu können (Jenewein et al. 2009; Dieker et al. 2013). Studien, die Gespräche zwischen Mensch und Computer sowie Mensch und Mensch verglichen, konnten zeigen, dass sich die Gesprächsführung der Teilnehmer kaum voneinander unterscheidet (Heyselaar et al. 2017). Daher ist der immer häufigere Einsatz von virtuellen Charakteren als Trainingsformat zur Verbesserung von kommunikativen Fähigkeiten nicht verwunderlich (für einen ausführlichen Überblick: Talbot 2019).

Das vom Bundesministerium für Bildung und Forschung (BMBF) seit 2018 geförderte Forschungsprojekt „Vicontact" (https://www.empirische-bildungsforschung-bmbf.de/ de/2372.php) versucht in diesem Sinne ebenfalls, feedbackgestützte Trainingsgespräche in einer virtuellen Umgebung zu erproben. Dabei entwickeln und evaluieren Experten der Europa-Universität Flensburg (EUF), der Psychologischen Hochschule Berlin (PHB) und der Schwerpunktprofessur für Forensische Psychiatrie und Psychotherapie der Universitätsmedizin Göttingen (UMG) eine VR-Anwendung für die Lehrerausbildung. Dabei sollen angehende Lehrer mithilfe einer Kombination aus VR-Training und Psychoedukation auf Gespräche mit Schülern vorbereitet werden, bei denen der Verdacht auf das Vorliegen eines sexuellen Missbrauchs besteht. Erste Erfahrungen mit forensischen Befragungen von Kindern durch den Einsatz virtueller Kinderfiguren machten bereits Pompedda et al. (2014, 2017; Krause et al. 2017). Dafür entwickelten sie 2-dimensionale Avatare, die 4- bzw. 6-jährigen Kindern nachempfunden waren und auf einem Computermonitor präsentiert wurden. Die Avatare hatten ein vorprogrammiertes Gedächtnis, welches entweder einen Missbrauchsfall oder keinen Missbrauchsfall beinhaltete, und aus narrativen Antwortsätzen bestand. Die Aufgabe des Interviewers bestand darin, durch den Einsatz gewünschter (z. B. offener) anstatt unerwünschter (z. B. geschlossener) Fragen, möglichst viele inhaltlich relevante und korrekte Informationen zu erfragen und zu einer richtigen Schlussfolgerung über den tatsächlichen Gedächtnisinhalt des Avatars zu kommen. Die Arbeitsgruppe konnte zeigen, dass bereits ein einstündiges Interviewtraining zu einer signifikanten Verbesserung des Interviewverhaltens geführt hat. Dieser Effekt trat jedoch nur in Kombination mit einem während des Trainings erhaltenen Feedbacks, welches den Probanden nach jedem Trainingsgespräch eine Beurteilung ihrer Fragetechniken vermittelte, ein (Pompedda et al. 2014).

Die Aufgabe der Probanden in Vicontact besteht darin, durch den Einsatz von erwünschten im Gegensatz zu unerwünschten Fragen herauszufinden, ob, und wenn ja, was dem Kind zugestoßen ist. Dabei werden 3 unterschiedliche Ereignisbedingungen unterschieden: sexueller Missbrauch, anderes interventionsbedürftiges Ereignis und andere Belastung. Die virtuelle Umgebung enthält ein 3-dimensionales Modell eines Klassenzimmers sowie eines von insgesamt 8 verschiedenen 3-dimensionalen und animierten virtuellen Kindern zwischen 10 und 12 Jahren (4 weiblich, 4 männlich). Die virtuelle Umgebung wird über eine HMD dargestellt (HTC Vive Pro, HTC Corporation, Taoyu- 
an City 330, Taiwan [R.O.C]). Da die Probanden natürliche Sprache verwenden sollen, wurde ein Spracherkennungsmodul integriert (Kaldi, Povey et al. 2011; Milde und Köhn 2018), welches in Echtzeit die Fragen in Text transkribiert. Das textbasiertes Dialog-Management(oder Chatbot)-System Chatscript (Wilcox und Wilcox 2013) wird verwendet, um die transkribierte Frage zu analysieren und eine inhaltlich adäquate Antwort aus dem jeweiligen Kindergedächtnis zu geben. Chatscript wurde bereits erfolgreich als Basis von VSP zum Training von Medizinstudenten eingesetzt und sorgt bei einer umfangreichen Implementierung von Frage-Antwort-Paarungen und einer damit verbundenen besseren Analyse von bestimmten Fragekonstellationen für eine hohe Rate an adäquaten Antworten (Maicher et al. 2017, 2019). Häufige unpassende Antworten seitens der VSP können den Eindruck eines unrealistischen Antwortverhaltens erwecken (Johnsen et al. 2005) und damit auf Kosten des sozialen Präsenzerlebens gehen. Durch die Kombination aus HMD und Chatbot-Technologien sind daher ein hohes Maß an Immersion auf der einen Seite und eine realistische Gesprächsführung auf der anderen Seite gegeben. Darüber hinaus erhalten die Probanden ein automatisiertes, computergeneriertes visuelles Feedback, welches nach jedem Gespräch u. a. positive sowie negative Beispiele eingesetzter Fragen zeigt.

In einer ersten Machbarkeitsstudie untersucht das Forschungsprojekt (geplante Stichprobe von $N=200$ ) Lehramtsstudenten anhand eines pseudorandomisierten experimentellen Designs, inwieweit ein VR-Training die Lerneffekte auf Verhaltensebene zusätzlich zu einer klassisch dargebotenen Psychoedukation verbessern kann. Erste belastbare Ergebnisse sind Mitte 2021 zu erwarten. Die VRUmgebung, einschließlich der technischen Umsetzung und der Psychoedukation, werden als „Open-source“-Projekt nach Abschluss der Studie allen interessierten Forschern und Praktikern frei zur Verfügung gestellt, solange eine wirtschaftliche Nutzung ausgeschlossen ist.

Die Ergebnisse der Studie könnten nicht nur Hinweise auf das Potenzial von VR und VSP in Kombination mit Chatbot-Technologien für die Lehrerausbildung geben, sondern auch wegweisend für die Anwendung in der forensischen Psychiatrie und Psychologie sein, wie beispielsweise der Ausbildung forensisch-psychiatrischen Gutachtern, psychologischen Gutachtern oder polizeilichen Ermittlern.

\section{Möglichkeiten, Grenzen und ethische Richtlinien für VR-Anwendungen}

Virtuelle-Realitäts-Anwendungen besitzen ein hohes Potenzial für die Forschung, Diagnostik und Therapie sowie Ausbildung von Fachpersonal im forensisch-psychiatrischen Bereich. Wie die vorgestellten Forschungsbeispiele gezeigt haben, ist eine große Stärke von VR, dass Situationen oder Trainingsbedingungen geschaffen werden können, die im Labor schwer kontrollierbar, ethisch nichtvertretbar oder überhaupt nicht umsetzbar sind. Dadurch ergibt sich eine Vielzahl neuer Forschungs- und Therapieszenarien, in denen sich VR-Anwendungen einsetzen lassen könnten. Dennoch ist festzuhalten, dass nur wenige Ansätze, wie die Diagnostik und Therapie von Phobien in der VR, eine stabile Befundlage haben, sodass die meisten Studien (insbesondere auch im forensisch-psychiatrischen Kontext) noch explorativer Natur sind. Das ist nachvollziehbar, wenn man bedenkt, dass erst in den letzten 10 Jahren die VRTechnik ihre gröbsten Kinderkrankheiten abgelegt hat und damit für ein breiteres Forscherfeld, insbesondere aber auch für vulnerable Personen, wie dies beispielsweise psychisch kranke Menschen darstellen, zugänglich wird. Umso mehr gilt es für zukünftige Studien zu klären, in welchen klinischen Kontexten VR, verglichen mit gängigen Verfahren, einen Mehrwert besitzt, welche Eigenschaften der VR einen Einfluss auf das Erleben und die Reaktion der Probanden haben (z.B. Präsentationsdauer, Stimulusqualität, Präsentationsform) und wie unterschiedliche Probandengruppen auf die VR reagieren. Dadurch kann eine solide empirische Grundlage für die Weiterentwicklung klinischer VRAnwendungen geschaffen werden.

Aus ökonomischer Perspektive gibt es heute immer weniger Gründe, die gegen den Einsatz von VR für Forschung, Diagnostik und Therapie sprechen: VR-Technologie, allen voran die HMD, wird immer günstiger, leichter zu bedienen trotz höherer technischer Komplexität, sodass anfängliche technisch-bedingte Schwierigkeiten wie Simulatorübelkeit in klinischen Anwendungen kaum noch auftreten (Bouchard und Rizzo 2019) und, durch neue kabellose HMD-Modelle, noch flexibler einsetzbar sind. Gleichzeitig werden VR-Entwicklungsumgebungen immer populärer und haben mittlerweile eine breite Onlinegemeinde, die durch ihren Support auch weniger technikaffinen Forschern den Einstieg erleichtern können. Die Entwicklung von VRAnwendungen ist dennoch mit einem nicht zu unterschätzenden Aufwand verbunden, da der Einsatz von VR-Anwendungen ein, im Vergleich zu vielen traditionelleren Untersuchungs- und Interventionsmethoden, erheblich größeres $\mathrm{Ma} \beta$ an technischer Komplexität mit sich bringt. Betrachtet man sich jedoch die visuelle Qualität der hier beispielhaft vorgestellten aktuellen Studien, so zeigt sich, dass keine spielfilmreifen Maßstäbe nötig sind, um immersive und Präsenz erzeugende virtuelle Umgebungen zu entwickeln.

Abschließend muss bei der Entwicklung und dem Einsatz von VR-Anwendungen auf die Einhaltung ethischer Richtlinien hingewiesen werden. Obwohl die Technik schon seit Längerem etabliert ist, wurden solche Richtlinien erst neuerdings zusammengestellt (für die allgemeine Anwen- 
dung in der Forschung: Madary und Metzinger 2016; für die spezifische Anwendung im forensisch-psychiatrischen Kontext: Fromberger et al. 2018a). Die Autoren geben zu bedenken, dass Ergebnisse der VR-Forschung und -Anwendungen auch missbraucht werden können (z.B. dass sexuelle Missbrauchstäter VR-Anwendungen zur Stimulation benutzen), sodass solche Szenarien mitbedacht werden sollten. Die Teilnahme an VR-Studien und VR-Therapien sollte gegenüber den Probanden hinsichtlich des potenziellen Nutzens und ihrer möglichen Nebenwirkungen transparent gestaltet werden. Darüber hinaus sollte stärker auf Datenschutz geachtet werden, da der Großteil der VR-Technologie abhängig von einer Internetanbindung ist. Darüber könnten Hersteller auf z. B. Bewegungsdaten der HMD zugreifen und individuelle Bewegungsprofile erstellen. Deshalb ist aus klinischer Anwenderperspektive sicherzustellen, dass die Speicherung und der Umgang mit Probandenund Patientendaten klinischen Sicherheitsstandards entsprechen. Abschließend weisen die Autoren darauf hin, offen und transparent mit Forschungsergebnissen, positiven sowie negativen, umzugehen.

Funding Open Access funding provided by Projekt DEAL.

Interessenkonflikt H. Barbe, B. Siegel, J.L. Müller und P. Fromberger geben an, dass kein Interessenkonflikt besteht.

Open Access Dieser Artikel wird unter der Creative Commons Namensnennung 4.0 International Lizenz veröffentlicht, welche die Nutzung, Vervielfältigung, Bearbeitung, Verbreitung und Wiedergabe in jeglichem Medium und Format erlaubt, sofern Sie den/die ursprünglichen Autor(en) und die Quelle ordnungsgemäß nennen, einen Link zur Creative Commons Lizenz beifügen und angeben, ob Änderungen vorgenommen wurden.

Die in diesem Artikel enthaltenen Bilder und sonstiges Drittmaterial unterliegen ebenfalls der genannten Creative Commons Lizenz, sofern sich aus der Abbildungslegende nichts anderes ergibt. Sofern das betreffende Material nicht unter der genannten Creative Commons Lizenz steht und die betreffende Handlung nicht nach gesetzlichen Vorschriften erlaubt ist, ist für die oben aufgeführten Weiterverwendungen des Materials die Einwilligung des jeweiligen Rechteinhabers einzuholen.

Weitere Details zur Lizenz entnehmen Sie bitte der Lizenzinformation auf http://creativecommons.org/licenses/by/4.0/deed.de.

\section{Literatur}

Alsina-Jurnet I, Gutiérrez-Maldonado J, Rangel-Gómez M-V (2011) The role of presence in the level of anxiety experienced in clinical virtual environments. Comput Human Behav 27:504-512. https:// doi.org/10.1016/j.chb.2010.09.018

Artstein R, Gandhe S, Leuski A, Traum D (2008) Field testing of an interactive question-answering character $\mathrm{Bd} .36$

Bailenson J (2018) Experience on demand: what virtual reality is, how it works, and what it can do. W. W. Norton \& Company. New York

Benbouriche M, Nolet K, Trottier D, Renaud P (2014) Virtual reality applications in forensic psychiatry. ACM Press, New York, New York, S 1-4
Benedict N (2010) Virtual patients and problem-based learning in advanced therapeutics. Am J Pharm Educ. https://doi.org/10.5688/ aj7408143

Bohil CJ, Alicea B, Biocca FA (2011) Virtual reality in neuroscience research and therapy. Nat Rev Neurosci 12:752-762. https://doi. org/10.1038/nrn3122

Botella C, Fernández-Álvarez J, Guillén V et al (2017) Recent progress in virtual reality exposure therapy for phobias: a systematic review. Curr Psychiatry Rep 19:42

Bouchard S, Rizzo AS (2019) Applications of virtual reality in clinical psychology and clinical cognitive neuroscience-an introduction. In: Virtual reality for psychological and Neurocognitive interventions, 1. Aufl. Springer, New York, S 1-13

Carl E, Stein AT, Levihn-Coon A et al (2019) Virtual reality exposure therapy for anxiety and related disorders: a meta-analysis of randomized controlled trials. J Anxiety Disord 61:27-36

Cho S, Ku J, Park J et al (2008) Development and verification of an alcohol craving-induction tool using virtual reality: craving characteristics in social pressure situation. Cyberpsychol Behav 11:302-309. https://doi.org/10.1089/cpb.2007.0149

Clay F, Howett D, FitzGerald J et al (2020) Use of Immersive virtual reality in the assessment and treatment of alzheimer's disease: a systematic review. J Alzheimers Dis 75:23-43. https://doi.org/10. 3233/JAD-191218

Cornet LJM, Van Gelder J-L (2020) Virtual reality: a use case for criminal justice practice. Psychol Crime Law 5:1-17. https://doi.org/ 10.1080/1068316X.2019.1708357

Côté S, Bouchard S (2005) Documenting the efficacy of virtual reality exposure with psychophysiological and information processing measures. Appl Psychophysiol Biofeedback 30:217-232. https:// doi.org/10.1007/s10484-005-6379-x

Craske MG, Hermans D, Vervliet B (2018) State-of-the-art and future directions for extinction as a translational model for fear and anxiety. Phil Trans R Soc B 373:20170025

Dechant M, Trimpl S, Wolff C et al (2017) Potential of virtual reality as a diagnostic tool for social anxiety: a pilot study. Comput Human Behav 76:128-134

Dieker LA, Rodriguez JA, Lignugaris Kraft B et al (2013) The potential of simulated environments in teacher education. Teach Educ Special Educ 37:21-33. https://doi.org/10.1177/088840641351 2683

Freeman D, Reeve S, Robinson A et al (2017) Virtual reality in the assessment, understanding, and treatment of mental health disorders. Psychol Med 47:2393-2400

Fromberger P, Meyer S, Kempf C et al (2015) Virtual viewing time: the relationship between presence and sexual interest in androphilic and gynephilic men. PLoS ONE 10:e127156. https://doi.org/10. 1371/journal.pone.0127156

Fromberger P, Jordan K, Müller JL (2014) Anwendung virtueller Realitäten in der forensischen Psychiatrie. Nervenarzt 85:298-303. https://doi.org/10.1007/s00115-013-3904-7

Fromberger P, Jordan K, Müller JL (2018a) Virtual reality applications for diagnosis, risk assessment and therapy of child abusers. Behav Sci Law 36:235-244. https://doi.org/10.1002/bsl.2332

Fromberger P, Meyer S, Jordan K, Müller JL (2018b) Behavioral monitoring of sexual offenders against children in virtual risk situations: a feasibility study. Front Psychol 9:504. https://doi.org/10. 3389/fpsyg.2018.00224

Groom V, Bailenson JN, Nass C (2009) The influence of racial embodiment on racial bias in immersive virtual environments. Soc Influ 4:231-248

Heyselaar E, Hagoort P, Segaert K (2017) In dialogue with an avatar, language behavior is identical to dialogue with a human partner. Behav Res Methods 49:46-60. https://doi.org/10.3758/s13428015-0688-7

Hubal RC, Kizakevich PN, Guinn CI et al (2000) The virtual standardized patient. In: Medicine meets virtual reality, S 133-138 
Jenewein K, Haase A, Hundt D, Liefold S (2009) Lernen in virtueller Realität. Ein Forschungsdesign zur Evaluation von Wahrnehmung in unterschiedlichen virtuellen Systemen (Apostolopoulos, Nicolas [Hrsg]; Hoffmann, Harriet [Hrsg]; Mansmann, Veronika [Hrsg]; Schwill, Andreas [Hrsg]: E-Learning 2009 Lernen im digitalen Zeitalter Münster ; New York ; München ; Berlin : Waxmann 2009, S 302-312 - (Medien in der Wissenschaft; 51) 302-312)

Johnsen K, Dickerson R, Raij A et al (2005) Experiences in using immersive virtual characters to educate medical communication skills. IEEE Proc 2005:179-186

Klein Tuente S, Bogaerts S, Bulten E et al (2019) Virtual reality aggression prevention therapy (VRAPT) versus waiting list control for forensic psychiatric inpatients: a multicenter randomized controlled trial. SSRN 3487845.

Krause N, Pompedda F, Antfolk J et al (2017) The effects of feedback and reflection on the questioning style of untrained interviewers in simulated child sexual abuse interviews. Appl Cognit Psychol 31:187-198

Laforest M, Bouchard S, Crétu A-M, Mesly O (2016) Inducing an anxiety response using a contaminated Virtual environment: validation of a therapeutic tool for obsessive-compulsive disorder. Front ICT 3:18

Laws DR, Gress CL (2004) Seeing things differently: The viewing time alternative to penile plethysmography. Leg Criminol Psychol 9:183-196

Madary M, Metzinger TK (2016) Real virtuality: a code of ethical conduct. Recommendations for good scientific practice and the consumers of VR-technology. Front Robot AI 3:235. https://doi.org/ 10.3389/frobt.2016.00003

Maicher K, Danforth D, Price A et al (2017) Developing a conversational virtual standardized patient to enable students to practice history-taking skills. Simul Healthc 12:124-131. https://doi.org/ 10.1097/SIH.0000000000000195

Maicher KR, Zimmerman L, Wilcox B et al (2019) Using virtual standardized patients to accurately assess information gathering skills in medical students. Med Teach 41:1053-1059. https://doi.org/10. 1080/0142159X.2019.1616683

Milde B, Köhn A (2018) Open source automatic speech recognition for German. In: Speech communication 13th ITG-symposium. VDE, S 1-5, Oldenburg

Milgram P, Kishino F (1994) A taxonomy of mixed reality visual-displays. IEICE Trans Inf \& Syst E77D:1321-1329

Muhlberger A, Petrusek S, Herrmann MJ, Pauli P (2005) Biocyberpsychologie: Subjektiv und physiologische Reaktionen von Flugphobikern und Gesunden bei Exposition mit virtuellen Flugen. [Biocyberpsychology: Subjective and physiological reactions in flight phobics and normal subjects during flight simulations]. Z Klin Psychol Psychother Forsch Prax 34:133-143

Park MJ, Kim DJ, Lee U et al (2019) A literature overview of virtual reality (VR) in treatment of psychiatric disorders: recent advances and limitations. Front Psych 10:505. https://doi.org/10.3389/ fpsyt.2019.00505
Parsons TD, Gaggioli A, Riva G (2017) Virtual Reality for Research in Social Neuroscience. Brain Sci 7:42. https://doi.org/10.3390/ brainsci7040042

Pompedda F, Antfolk J, Zappalà A, Santtila P (2017) A combination of outcome and process feedback enhances performance in simulations of child sexual abuse interviews using avatars. Front Psychol 8:2062. https://doi.org/10.3389/fpsyg.2017.01474

Pompedda F, Zappalà A, Santtila P (2014) Simulations of child sexual abuse interviews using avatars paired with feedback improves interview quality. Psychol Crime Law 21:28-52. https://doi.org/10. 1080/1068316X.2014.915323

Povey D, Ghoshal A, Boulianne G et al (2011) The Kaldi speech recognition toolkit. IEEE 2011 Workshop on Automatic Speech Recognition and Understanding.

Reger GM, Holloway KM, Candy C et al (2011) Effectiveness of virtual reality exposure therapy for active duty soldiers in a military mental health clinic. J Traum Stress 24:93-96. https://doi.org/10. 1002/jts.20574

Renaud P, Chartier S, Rouleau J-L et al (2009) Gaze behavior nonlinear dynamics assessed in virtual immersion as a diagnostic index of sexual deviancy: preliminary results. J Virtual Real Broadcast. https://doi.org/10.20385/1860-2037/6.2009.3

Renaud P, Trottier D, Rouleau J-L et al (2014) Using immersive virtual reality and anatomically correct computer-generated characters in the forensic assessment of deviant sexual preferences. Virtual Real 18:37-47

Ryan WS, Cornick J, Blascovich J, Bailenson JN (2019) Virtual reality: whence, how and what for. In: Rizzo AS, Bouchard S (Hrsg) Virtual reality for psychological and Neurocognitive interventions. Springer, New York, S 15-46

Schuemie MJ, van der Straaten P, Krijn M, van der Mast CAPG (2004) Research on presence in virtual reality: a survey. Cyberpsychol Behav 4:183-201. https://doi.org/10.1089/109493101300117884

Seinfeld S, Arroyo-Palacios J, Iruretagoyena G et al (2018) Offenders become the victim in virtual reality: impact of changing perspective in domestic violence. Sci Rep 8:1-11

Slater M (2009) Place illusion and plausibility can lead to realistic behaviour in immersive virtual environments. Phil Trans R Soc B 364:3549-3557. https://doi.org/10.1098/rstb.2009.0138

Smeijers D, Koole SL (2019) Testing the effects of a virtual reality game for aggressive impulse management (VR-GAIME): study protocol. Front Psychiatry. https://doi.org/10.3389/fpsyt.2019. 00083

Talbot T (2019) Virtual human standardized patients for clinical training. In: Virtual reality for psychological and Neurocognitive interventions. Springer, New York, S 387-405

Welsch R, Castell C von, Rettenberger M, Turner D, Hecht H, Fromberger P (2020) Sexual attraction modulates interpersonal distance and approach-avoidance movements towards virtual agents in males. Plos One 15(4):e0231539. https://doi.org/10.1371/journal. pone. 0231539

Wilcox B, Wilcox S (2013) Making it real: Loebner-winning chatbot design. Arbor. https://doi.org/10.3989/arbor.2013.764n6009 